\title{
Vegetación arbórea del Cerro Tres Puntas de Pilasca, (Salas-Motupe), Lambayeque, Perú Arboreal vegetation of the Cerro Tres Puntas de Pilasca, (Salas-Motupe), Lambayeque, Perú
}

\author{
Guillermo E. Delgado-Paredes ${ }^{1,2^{*}}$, Cecilia Vásquez-Díaz ${ }^{2}$, Fernando Tesén-Núñez ${ }^{2}$, \\ Boris Esquerre-Ibañez ${ }^{2}$, Felipe Zuñe Da-Silva ${ }^{2}$ y Consuelo Rojas-Idrogo ${ }^{1,2}$
}

\begin{abstract}
:
From March to June, 2018 the seasonally dry forest Cerro Tres Puntas of Pilasca (Salas-Motupe, Lambayeque, Perú) was studied, in order to obtain information on its structure and its floristic composition. In a linear transect, about $11.4 \mathrm{ha}$, specimens of woody plants with $\mathrm{DBH} \geq 5.0 \mathrm{~cm}$ were assessed. 410 individuals representing 17 species, 17 genera and 10 families were recorded. The most abundant species were Vachellia macracantha (Fabaceae 154 individuals) and Celtis iguanaea (Cannabaceae 55 individuals). The families with the highest number of species were Fabaceae (7) and Malvaceae (2). The basimetric area was $343.86 \mathrm{~m}^{2}$ ha 1 highlighting Ficus obtusifolia with $139.23 \mathrm{~m}^{2} \mathrm{ha}^{-1}$ and Beilschmiedia sulcata with $120.90 \mathrm{~m}^{2} \mathrm{ha}^{-1}$. The Importance Value Index for the species reached the main values in F. obtusifolia (49.34), V. macracantha (46.75) and $B$. sulcata (41.57), while the Family Value Index was widely higher in the Fabaceae family (111.86). While these results show that this forest is a precarious version of the region's, $F$. obtusifolia individuals between 35-39.9 $\mathrm{m}$ high and 210-219.9 cm DBH were found. This study will help to accomplish an efficient conservation and reforestation program in order to improve the Cerro Tres Puntas forest, located in one of the poorest areas of the nation.
\end{abstract}

Key words: Basimetic area, seasonally dry forest, diametric distribution, floristic diversity, floristic structure, Lambayeque.

\section{Resumen:}

De marzo a junio de 2018 se estudió el bosque estacionalmente seco en Cerro Tres Puntas de Pilasca (SalasMotupe, Lambayeque, Perú) con la finalidad de obtener información sobre su estructura y composición florística. En un transecto lineal de 11.4 ha, aproximadamente, se evaluaron especímenes de plantas leñosas con DAP $\geq 5.0 \mathrm{~cm}$. Se registraron 410 individuos que representan a 17 especies, 17 géneros y 10 familias. Los taxones más abundantes fueron Vachellia macracantha (Fabaceae 154 individuos) y Celtis iguanaea (Cannabaceae 55 individuos) y las familias mejor representadas corespondieron a Fabaceae, con siete especies y Malvaceae, con dos. El área basal total fue de $343.86 \mathrm{~m}^{2} \mathrm{ha}^{-1}$, en la que destacan Ficus obtusifolia con 139.23 $\mathrm{m}^{2} \mathrm{ha}^{-1}$ y Beilschmiedia sulcata con $120.90 \mathrm{~m}^{2} \mathrm{ha}^{-1}$. El Índice de Valor de Importancia alcanzó los mayores valores en F. obtusifolia (49.34), V. macracantha (46.75) y C. ruizii (41.57); en tanto que el Índice de Valor de la Familia fue más alto para Fabaceae, con 111.86, seguida de Moraceae (46.74) y Lauraceae (45.33). Si bien, los resultados muestran que este bosque es una versión precaria de los existentes en la región, todavía se identificó la presencia de individuos de $F$. obtusifolia entre 35 - 39.9 m de altura y DAP de 210 a $219.9 \mathrm{~cm}$. El presente estudio contribuirá a desarrollar un programa de conservación, y de reforestación eficaz para mejorar el bosque del Cerro Tres Puntas, ubicado en una de las áreas más pobres del país.

Palabras clave: Área basal, bosque estacionalmente seco, distribución diamétrica, diversidad florística, estructura florística, Lambayeque.

\footnotetext{
${ }^{1}$ Facultad de Ciencias Biológicas, Universidad Nacional Pedro Ruiz Gallo, Lambayeque, Perú.

${ }^{2}$ Laboratorio General de Biotecnología, Universidad Nacional Pedro Ruiz Gallo, Lambayeque, Perú.

*Autor por correspondencia: guidelg2015@yahoo.es
} 


\section{Introducción}

Los bosques estacionalmente secos (BES) en el Neotrópico se distribuyen desde el norte de México hasta el sur de Brasil y conforman $66.7 \%$ de la superficie de este tipo de vegetación del mundo (Miles et al., 2006). En Perú, los BES son ecosistemas amenazados, poco estudiados y comprenden varios subtipos (Linares-Palomino, 2006; Linares-Palomino y Pennington, 2007). Los BES de montaña y de llanura de la costa norte de Perú son muy frágiles; se extienden desde la Península de Santa Elena, en el sur del Ecuador, hasta varias regiones del noroeste del Perú, entre las cuales están Tumbes, Piura, Lambayeque y el norte de La Libertad, así como el piso inferior del valle del Marañón. Las dos últimas áreas se comunican por medio del Paso de Porculla, aunque son unidades fitogeográficas distintas (Brack y Mendiola, 2004). También se le conoce como región de endemismos Tumbesina, la cual es compartida por Ecuador y Perú, y se le considera una de las zonas más importantes del mundo (Aguirre et al., 2006).

Algunos estudios han revelado el gran número de especies endémicas presentes en el BES del norte y noroeste del Perú, con alrededor de $26 \%$ de ellas diseminadas en varias comunidades, tales como Prosopis limensis Benth. (algarrobo) y Colicodendron scabridum Seem. (zapote) (Sagástegui et al., 1999). Pero, no solo los niveles de diversidad y endemismo se refieren a plantas, sino también a vertebrados como Tremarctos ornatus Cuvier, 1825 (oso de anteojos), Lagidium peruanum Meyen, 1833 (vizcacha), Penelope albipennis Taczanowski, 1878 (pava aliblanca) y Bothrops barnetti Parker, 1994 (macanche), entre otros (Aguilar, 1994).

Los trabajos sobre el BES, aunque no son muy numerosos,4 sí han resultado significativos; . son particularmente interesantes los realizados por Weberbauer (1922; 1930; 1936; 1945), quien aportó aspectos florísticos y fitogeográficos de la flora del Perú y elaboró un mapa de vegetación, hasta ahora insuperable, con énfasis en el BES. En la década pasada destacan la lista anotada de especies leñosas y formaciones vegetales en los BES de la región de endemismo Tumbesina de Ecuador y Perú (Aguirre et al., 2006), así como de las plantas leñosas como una nueva herramienta en internet para estudios taxonómicos, ecológicos y de biodiversidad (Linares-Palomino y Pennington, 2007). 
Con base en datos de inventarios forestales se analizaron y discutieron los patrones de estructura y diversidad de los BES de dos áreas de conservación de la Reserva de la Biosfera del Noroeste (Perú), el Parque Nacional Cerros de Amotape y la Zona Reservada de Tumbes (Leal-Pinedo y Linares-Palomino, 2005). Se abordaron, los patrones de distribución espacial de las especies arbóreas como Eriotheca ruizzii (K. Schum.) A. Robyns (pasayo), Bursera graveolens Triana \& Planch. (palo santo), Caesalpinia glabrata Kunth (charán) y Cochlospermum vitifolium (Willd.) Spreng. (polo polo) en un BES del Parque Nacional Cerros de Amotape, en el noroeste del Perú, (Linares-Palomino, 2005). En los alrededores de Jaén (Cajamarca, Perú), en relictos del bosque tropical estacionalmente seco, se han efectuado ocho inventarios sobre la vegetación leñosa, endemismos y su estado de conservación (Marcelo-Peña, 2008).

Por otro lado, con la finalidad de diseñar mapas y llevar a cabo la clasificación de vegetación en ecosistemas estacionales de los bosques secos de Piura (Perú), con latos de inventarios florísticos, se identificaron seis grupos de vegetación; de ellos, cinco coincidieron con los tipos de vegetación descritos hace 100 años por A. Weberbauer (Weberbauer, 1922; 1930; 1936; 1945) (La Torre-Cuadros y Linares-Palomino, 2008). En la provincia de Ayabaca (Piura), se evaluaron las características edáficas y la composición florística de los BES La Menta y Timbes (Rasal et al., 2011).

En la región Lambayeque (Perú), también se han hecho algunos estudios en el BES. En la Reserva Ecológica Chaparrí, que forma parte del punto candente de biodiversidad Tumbes-Chocó-Magdalena, se elaboró un patrón estructural e inventario florístico, del que resultó evidente un bosque bastante empobrecido, en relación con otros BES de la región (Linares-Palomino y Ponce-Álvarez, 2009). En esta misma Reserva Ecológica Privada Chaparrí se determinó la fenología (desarrollo vegetativo, floración y fructificación) de 17 especies vegetales de las que se alimenta pava aliblanca, especie endémica seriamente amenazada (Martos et al., 2009).

Con base en lo anterior, además de que los bosques naturales del Perú han sido escasamente estudiados, y que cada vez es mayor la presión antrópica que conlleva a su depredación; y que el cambio climático continúa alterando los patrones de distribución vegetal, entre otros factores (Steffen et al., 2011), se llevó acabo el presente trabajo, cuyo objetivo fue determinar la composición florística del BES del Cerro Tres Puntas con 
el propósito de facilitar la elaboración de planes de reforestación, conservación y utilización, para con ello contribuir al conocimiento de los ecosistemas naturales del Perú.

\section{Materiales y Métodos}

\section{Área de estudio}

El área de estudio se localiza entre los 6¹4'16.23" S - 79³5'34.81" O y los 6¹2'46.46" S 79³3'59.58" O; comprende el Cerro Tierra Blanca (1 986 msnm), el Cerro Gato (1 545 msnm), ambos pertenecientes al distrito de Salas, y el Cerro Tres Puntas (1 590 msnm), del distrito de Motupe, en la provincia y región de Lambayeque, Perú (Figuras 1a-b); estos tres cerros abarcan una extensión aproximada de 2975 ha.

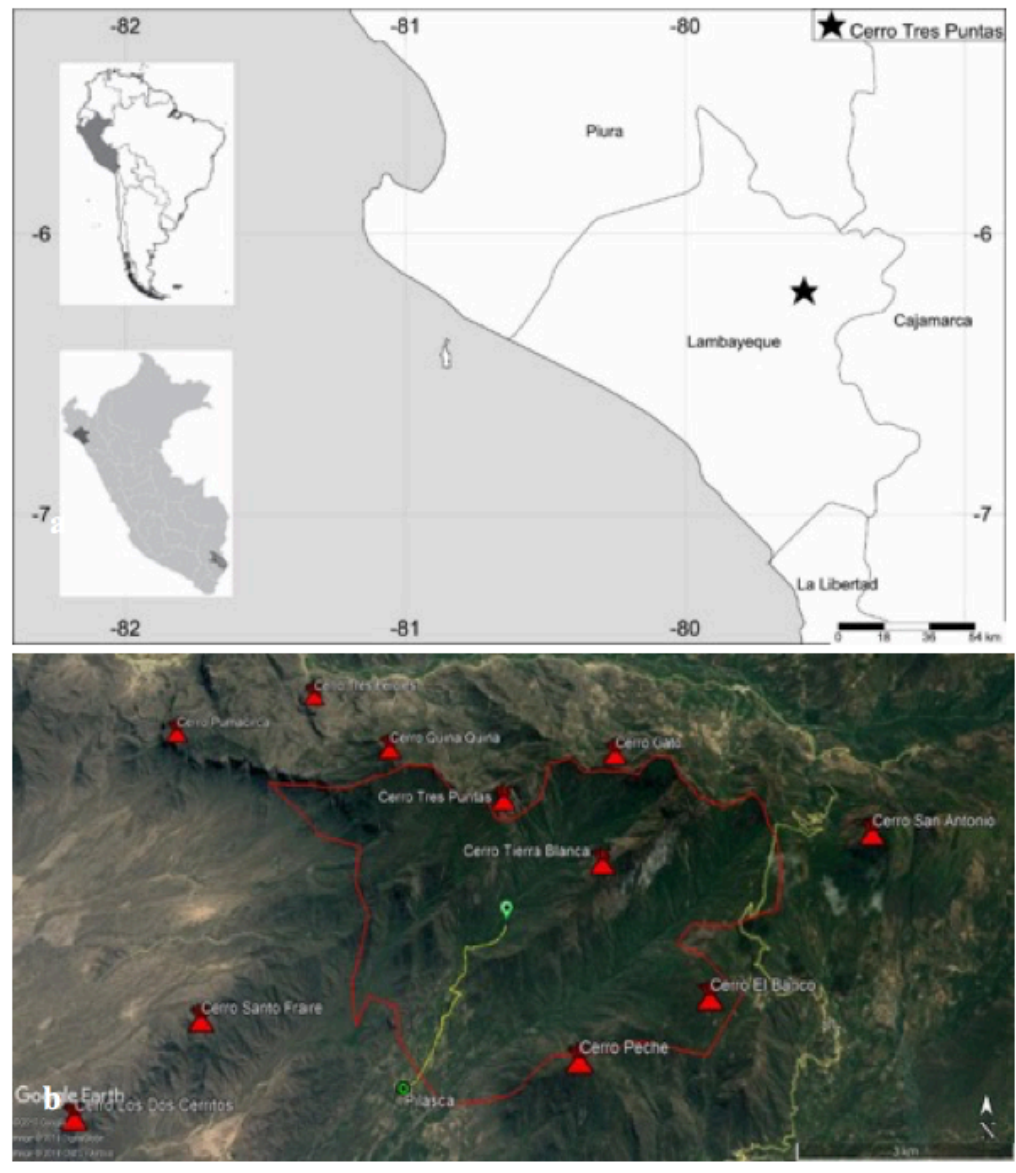

Fuente: Google Earth (2019).

Figura 1. a) Mapa de la región Lambayeque, indicando con la estrella la localidad de Pilasca, donde se ubica el Cerro Tres Puntas; b) Área que comprende los Cerros Tierra Blanca, Gato y Tres Puntas del BES (Salas-Motupe, Lambayeque, Perú). 
El caserío Pilasca es uno de los más pobres de Lambayeque (Gobierno Regional de Lambayeque, 2013) en el que, la agricultura de maíz (Zea mays L.), yuca (Manihot esculenta (rantz) y frutales, así como la ganadería de vacunos y caprinos son de subsistencia; ocupa pequeños claros del bosque, donde no hay selección de semillas, fertilización, ni control de plagas y enfermedades. Los comuneros de Pilasca han utilizado las especies del bosque desde tiempos ancestrales para la fabricación de muebles, construcción de viviendas rústicas y corrales, al igual que la leña como combustible; razón por la cual Loxopterygium huasango Spruce ex Engl. (hualtaco), Handroanthus chrysanthus (Jacq.) S. O. Grose (guayacán amarillo), Colicodendron scabridum (Kunth) Seem. (zapote), Prosopis limensis Benth. (algarrobo) y Cedrela sp. (cedro), prácticamente ya no existen.

En el aspecto geológico, la zona corresponde a la unidad litoestratigráfica Grupo Salas (Oi) del Paleozoico o Formación Salas, (Pi-s) Ordovícico inferior (480 a 470 millones de años) (Gobierno Regional de Lambayeque, 2013; López, 2013).

El área en la que se hizo la investigación es un ecosistema de bosque semidenso estacionalmente seco de colina y montaña (Bes-cm) (Minam, 2019). El levantamiento de los datos se realizó en un lugar ubicado entre los 373 y los 720 msnm; el punto extremo A corresponde al ingreso del caserío Pilasca $(373 \mathrm{~m})$, donde se inició la evaluación, hasta el punto extremo E (720 msnm), en el que terminó, cuya topografía es agreste con enormes peñascos y quebradas profundas, secas y escasos claros pedregosos. Los datos de temperatura, humedad relativa y precipitación correspondieron a los de la Estación CO-Meteorológica Jayanca (LA Vic) (78 msnm), la más cercana al Cerro Tres Puntas de Pilasca, de la que se registraron los siguientes datos: temperatura máxima y mínima y humedad relativa de marzo $2018,34.0$ y $19.0{ }^{\circ} \mathrm{C}$ y $78.4 \%$, respectivamente; mientras que, en junio $2018,26.2$ y $15.0{ }^{\circ} \mathrm{C}$ y $79.1 \%$, respectivamente. En los cuatro meses de evaluación, la precipitación fue $0.0 \mathrm{~mm} \mathrm{día}{ }^{-1}$ (Senamhi, 2018). 


\section{Muestreo}

El trabajo de campo se llevó a cabo de marzo a junio de 2018 y se utilizó el método propuesto por Gentry $(1982$; 1995a) para el muestreo de plantas leñosas, con sustanciales modificaciones, debido a la difícil y peligrosa geografía del terreno. Se aplicó un muestreo lineal, cuya línea base fue una trocha (camino estrecho o brecha) relativamente segura que los lugareños utilizan para acceder, ocasionalmente, a la cima del Cerro Tres Puntas (Figura 2).

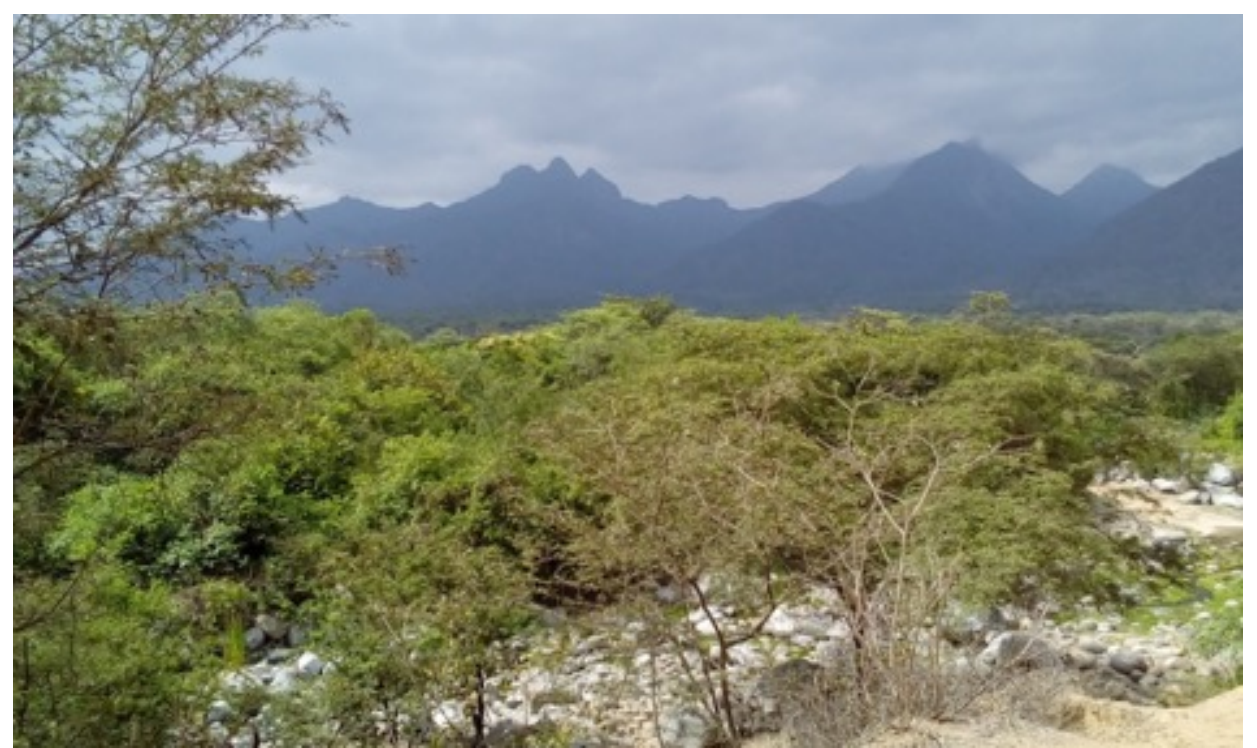

Figura 2. Área de estudio en la que se muestra el Cerro Tres Puntas (SalasMotupe, Lambayeque, Perú).

Se establecieron cuatro unidades de muestreo en una extensión total de $3801 \mathrm{~m}$, designadas con los puntos A (373 msnm), B (456 msnm), B-C (565 msnm), C-D (677 msnm) y D-E (720 msnm), que abarcaron $958 \mathrm{~m}, 961 \mathrm{~m}, 968 \mathrm{~m}$ y $914 \mathrm{~m}$, respectivamente. Del centro de la trocha, con un GPS Garmin 60CSX y una cinta métrica, se midieron $15 \mathrm{~m}$ a la derecha y $15 \mathrm{~m}$ a la izquierda, para un total de $30 \mathrm{~m}$, lo que permitió evaluar alrededor de $114030 \mathrm{~m}^{2}$. Se registraron todos los individuos leñosos de DAP $(1.3 \mathrm{~m}$ de la base del tronco $) \geq 5.0 \mathrm{~cm}$.

Para individuos con troncos ramificados, se midió el diámetro de cada ramificación a una altura de $1.3 \mathrm{~m}$ de la base del tronco, con una cinta diamétrica Forestry Suppliers, Inc. (Modelo 283D/5M). Como las especies evaluadas eran conocidas, solamente, se 
colectaron muestras botánicas para su registro y depósito en el Herbario de la Universidad Nacional Pedro Ruiz Gallo de Lambayeque (Herbario HPR). Asimismo, se prensaron ejemplares herbáceos, presentes en el área de colecta, para su posterior identificación taxonómica. Para las familias, se siguieron los postulados filogenéticos del Grupo de Filogenia de las Angiospermas (APG, 2009; 2016).

\section{Procesamiento y análisis de la información}

Para determinar la abundancia o densidad de los taxones muestreados se utilizó la clasificación de Maleheiros y Rotta (1982), la cual los cataloga como raros o escasos ( 1 - 6 individuos); poco abundantes (7 - 25); y abundantes (más de 25 individuos). La estructura horizontal del bosque se describió a partir de la distribución del número de árboles por clase diamétrica y de altura (Hawley y Smith, 1972). Los análisis fitosociológicos se hicieron por medio del freeware FITOPAC 2.1 (Shepherd, 2010). Con dicho soporte, se calcularon los siguientes parámetros: Densidad Relativa (DER), Frecuencia Relativa (FER), Área Basal Relativa ( $A B$ ) o Cobertura Relativa (COB), Índice de Valor de Importancia (IVI) y Valor de Importancia para la Familia (VIF); fórmulas que fueron descritas por Rangel y Velásquez (1997).

\section{Resultados}

\section{Composición florística}

En los $114030 \mathrm{~m}^{2}$ estudiados se inventariaron 410 individuos de árboles y arbustos con $\mathrm{DAP} \geq 5.0 \mathrm{~cm}$ que correspondieron a 17 especies, 17 géneros y 10 familias, registrados en 47 unidades de muestreo (Cuadro 1). A los taxa arbóreos y arbustivos (Cuadro 1 ) se sumaron numerosas herbáceas con diferentes hábitos de crecimiento como bejuco, enredadera, epífita, subarbusto, sufrútice, entre otros; colectadas con flores y frutos en mayo de 2018, entre las que pueden citarse: Salvia occidentalis Sw. (Lamiaceae), Psittacanthus chanduyensis Eichler (Loranthaceae), Croton pavonis Müll. Arg. (Euphorbiaceae), Scutia spicata (Wild.) Weberb. (Rhamnaceae), Alternanthera pubiflora Kuntze (Amaranthaceae), 
Dalechampia scandens L. (Euphorbiaceae), Cordia macrocephala (Desv.) Kunth (Boraginaceae), Plumbago scandens L. (Plumbaginaceae), Heliotropium angiospermum Murray (Boraginaceae), Waltheria ovata Cav. (Malvaceae), Wedelia latifolia DC. (Asteraceae), Cardiospermum corindum L. (Sapindaceae), Tessaria integrifolia Ruiz \& Pav. (Asteraceae), Pseudogynoxys cordifolia (Cass.) Cabrera (Asteraceae), Tetramerium nervosum Nees (Malvaceae), Scoparia dulcis L. (Scrophulariaceae) y Cestrum auriculatum L'Hér. (Solanaceae). 
Cuadro 1. Principales características dasométricas de las especies presentes en el área evaluada del BES Cerro Tres Puntas (Salas-Motupe, Lambayeque, Perú).

\begin{tabular}{|c|c|c|c|c|c|c|c|}
\hline Familia/Especie & Nombre común & Háb. crec. & Núm. indiv./\% & $\begin{array}{l}\text { Unidad } \\
\text { muestr. }\end{array}$ & $\begin{array}{l}\text { DAP } \\
(\mathrm{cm})\end{array}$ & $\begin{array}{c}\text { Altura total } \\
\text { (m) }\end{array}$ & $\begin{array}{c}\text { Diám. copa } \\
\text { (m) }\end{array}$ \\
\hline \multicolumn{8}{|l|}{ Anacardiaceae } \\
\hline Loxopterygium huasango Spruce ex Engl. & Hualtaco & Árbol & $20 / 4.9$ & 4 & 27.79 & 12.83 & 8.84 \\
\hline \multicolumn{8}{|l|}{ Boraginaceae } \\
\hline Cordia lutea Lam. & Overo & Árbol/ Arbust. & $29 / 7.1$ & 3 & 1.83 & 5.97 & 6.52 \\
\hline \multicolumn{8}{|l|}{ Burseraceae } \\
\hline Bursera graveolens (Kunth) Triana \& Planch. & Palo santo & Árbol & $4 / 1.0$ & 2 & 22.45 & 11.00 & 6.88 \\
\hline \multicolumn{8}{|l|}{ Fabaceae } \\
\hline Albizia multiflora (Kunth) Barneby \& J.W. Grimes & Angolo & Árbol & $8 / 2.0$ & 4 & 33.39 & 14.75 & 11.43 \\
\hline Caesalpinia glabrata Kunth & Charán & Árbol & $5 / 1.2$ & 1 & 11.92 & 7.00 & 6.92 \\
\hline Erythrina smithiana Krukoff & Frejolillo & Árbol & $32 / 7,8$ & 3 & 36.03 & 11.69 & 9.14 \\
\hline Pithecellobium excelsum (Kunth) Mart. & Chaquiro & Árbol/ Arbust. & $21 / 5.1$ & 4 & 11.04 & 7.33 & 9.64 \\
\hline Prosopis limensis Benth. & Algarrobo & Árbol & $9 / 2.2$ & 1 & 37.31 & 16.56 & 12.89 \\
\hline Senna atomaria (L.) Irwing \& Barneby & Chapa & Árbol & $15 / 3.7$ & 2 & 9.83 & 5.47 & 4.23 \\
\hline
\end{tabular}




\begin{tabular}{|c|c|c|c|c|c|c|c|}
\hline Vachellia macracantha (Willd.) Seigler \& Ebinger & Faique & Árbol/ Arbust. & $154 / 37.6$ & 4 & 16.34 & 8.54 & 8.99 \\
\hline \multicolumn{8}{|l|}{ Lauraceae } \\
\hline Beilschmiedia sulcata (Ruiz \& Pav.) Kosterm & Palta de zorro & Árbol & $1 / 0.2$ & 1 & 124.10 & 12.00 & 22.10 \\
\hline \multicolumn{8}{|l|}{ Malvaceae } \\
\hline Eriotheca ruizii (K. Schum.) A. Robyns & Pasayo & Árbol & $6 / 1.5$ & 1 & 31.3 & 10.58 & 7.37 \\
\hline Guazuma ulmifolia Lam. & Guazumo & Árbol & $11 / 2.7$ & 3 & 27.42 & 12.83 & 10.52 \\
\hline \multicolumn{8}{|l|}{ Moraceae } \\
\hline Ficus obtusifolia Kunth & Higuerón & Árbol & $17 / 4.2$ & 4 & 120.06 & 19.35 & 24.00 \\
\hline \multicolumn{8}{|l|}{ Olacaceae } \\
\hline Ximenia americana L. & Ciruelillo & Árbol & $17 / 4.2$ & 3 & 11.40 & 10.06 & 5.9 \\
\hline \multicolumn{8}{|l|}{ Sapindaceae } \\
\hline Sapindus saponaria L. & Choloque & Árbol & $6 / 1.5$ & 3 & 17.6 & 10.00 & 6.45 \\
\hline \multicolumn{8}{|l|}{ Cannabaceae } \\
\hline Celtis iguanaea (Jacq.) Sarg. & Palo blanco & Árbol & $55 / 13.4$ & 4 & 38.48 & 14.62 & 12.99 \\
\hline Total & & & 410 & 47 & 26.20 & 10.45 & 9.81 \\
\hline
\end{tabular}

Háb. crec. = Hábito de crecimiento; Núm. indiv. = Número de individuos; Unidad muestr. = Unidad de muestreo; DAP = Diámetro a la Altura del Pecho; Diám. copa = Diámetro de copa 


\section{Estructura}

De las 17 especies inventariadas, pocos ejemplares de Cordia lutea Lam., Vachellia macracantha (Willd.) Seigler \& Ebinger y Pitecellobium excelsum (Kunth) Benth. mostraron hábito de crecimiento arbustivo con más de 10 fustes por individuo, en algunos casos. Del total de especies registradas, solo seis [L. huasango, V. macracantha, P. excelsum, Albizia multiflora (Kunth) Barneby \& J.W. Grimes, Ficus obtusifolia Kunth y Celtis iguanaea (Jacq.) Sarg.] se registraron en las cuatro unidades de muestreo; en tanto que, Caesalpinia glabrata Kunth, P. limensis, E. ruizii y Beilschmiedia sulcata (Ruiz $\&$ Pav.) Kosterm. solamente se identificaron en una de ellas.

El mayor número de los 410 individuos inventariados correspondió a la tercera unidad de muestreo (565 - $677 \mathrm{msnm}$ ), con 194 (47.32\%); lo que se atribuye a lo agreste y pedregoso del territorio, donde no es posible desarrollar actividades agropecuarias; en contraste, en la segunda unidad de muestreo (456 - $565 \mathrm{msnm}$ ) el número de individuos fue menor con $42(10.24 \%)$, esto porque es el área con mayor vocación agrícola, por ello el bosque ha sido fuertemente depredado (Cuadro 2). 
Cuadro 2. Distribución de individuos por unidad de muestreo de las especies presentes en el área evaluada del BES Cerro Tres Puntas (Salas-Motupe, Lambayeque, Perú).

\begin{tabular}{|c|c|c|c|c|c|c|c|c|}
\hline \multirow{3}{*}{ Especie } & \multicolumn{8}{|c|}{ Unidades de muestreo } \\
\hline & \multicolumn{2}{|c|}{1} & \multicolumn{2}{|c|}{2} & \multicolumn{2}{|c|}{3} & \multicolumn{2}{|c|}{4} \\
\hline & $\mathbf{N}^{\circ}$ & $\%$ & $\mathbf{N}^{\circ}$ & $\%$ & $\mathbf{N}^{\circ}$ & $\%$ & $\mathbf{N}^{\circ}$ & $\%$ \\
\hline Beilschmiedia sulcata (Ruiz \& Pav.) Kosterm & 0 & 0.0 & 0 & 0.0 & 0 & 0.0 & 1 & 1.56 \\
\hline Bursera graveolens Triana \& Planch. & 2 & 1.82 & 0 & 0.0 & 2 & 1.03 & 0 & 0.0 \\
\hline Caesalpinia glabrata Kunth & 0 & 0.0 & 0 & 0.0 & 5 & 2.58 & 0 & 0.0 \\
\hline Celtis iguanaea (Jacq.) Sarg. & 9 & 8.18 & 7 & 16.67 & 23 & 11.86 & 16 & 25.00 \\
\hline Cordia Iutea Lam. & 10 & 9.10 & 2 & 4.76 & 17 & 8.76 & 0 & 0.0 \\
\hline Erythrina smithiana Krukoff & 0 & 0.0 & 1 & 2.38 & 26 & 13.40 & 5 & 7.81 \\
\hline Eriotheca ruizii (K. Schum.) A. Robyns & 0 & 0.0 & 0 & 0.0 & 6 & 3.09 & 0 & 0.0 \\
\hline Ficus obtusifolia Kunth & 1 & 0.91 & 1 & 2.38 & 3 & 1.55 & 12 & 18.75 \\
\hline Guazuma ulmifolia Lam. & 4 & 3.64 & 5 & 11.90 & 2 & 1.03 & 0 & 0.0 \\
\hline Loxopterygium huasango Spruce ex Engl. & 2 & 1.82 & 4 & 9.52 & 12 & 6.19 & 2 & 3.13 \\
\hline Pithecellobium excelsum (Kunth) Mart. & 11 & 10.0 & 4 & 9.52 & 5 & 2.58 & 1 & 1.56 \\
\hline Albizia multiflora (Kunth) Barneby \& J.W. Grimes & 2 & 1.82 & 2 & 4.76 & 2 & 1.03 & 2 & 3.13 \\
\hline Prosopis limensis Benth. & 9 & 8.18 & 0 & 0,0 & 0 & 0.0 & 0 & 0.0 \\
\hline Sapindus saponaria L. & 1 & 0.91 & 3 & 7.14 & 2 & 1.03 & 0 & 0.0 \\
\hline Senna atomaria (L.) Irwing \& Barneby & 9 & 8.18 & 6 & 14.29 & 0 & 0.0 & 0 & 0.0 \\
\hline Vachellia macracantha (Willd.) Seigler \& Ebinger & 47 & 42.73 & 7 & 16.67 & 81 & 41.75 & 19 & 29.69 \\
\hline Ximenia americana L. & 3 & 2.73 & 0 & 0.0 & 8 & 4.12 & 6 & 9.38 \\
\hline Total $=410$ individuos & 110 & 26.83 & 42 & 10.24 & 194 & 47.32 & 64 & 15.61 \\
\hline
\end{tabular}


El dosel superior se conformó por árboles dominantes de $F$. obtusifolia y $P$. limensis, aunque con individuos muy dispersos. Los ejemplares de mayor tamaño fueron $F$. obtusifolia con $19.35 \mathrm{~m}$ de altura, seguidos por $P$. limensis (16.56 m), P. excelsum (14.75 $\mathrm{m})$ y $C$. iguanaea, $(14.62 \mathrm{~m}$ ) en una distribución de las alturas que no es del todo una "J" invertida, como se espera en bosques naturales y en bosques regulares.

El diámetro de copa fue mayor en $F$. obtusifolia $(24.00 \mathrm{~m})$ y B. sulcata $(22.10 \mathrm{~m})$ (Cuadro 3). De igual manera, en la estructura horizontal del bosque se determinó que en el transecto 4 se presentaron los individuos con más altura (13.59 m) y diámetro, $(48.99 \mathrm{~cm})$, lo que es muy superior al diámetro registrado en el transecto 1, con $20.11 \mathrm{~cm}$, que corresponde a la mitad de su tamaño (Cuadro 4).

Cuadro 3. Distribución de individuos por clase diamétrica y altimétrica, densidad de las especies presentes en el área evaluada del BES Cerro Tres Puntas (SalasMotupe, Lambayeque, Perú).

\begin{tabular}{|c|c|c|c|c|c|}
\hline $\begin{array}{l}\text { Clase diamétrica } \\
(\mathrm{cm})\end{array}$ & $\begin{array}{l}\text { Individuos } \\
\text { (Número) }\end{array}$ & $\begin{array}{c}\text { Clase } \\
\text { altimétrica }(\mathrm{m})\end{array}$ & $\begin{array}{l}\text { Individuos } \\
\text { (Número) }\end{array}$ & Densidad & $\begin{array}{l}\text { Especies } \\
\text { (Número) }\end{array}$ \\
\hline $5-9.9$ & 86 & $0-4.9$ & 9 & Abundante & 4 \\
\hline $10-19.9$ & 128 & $5-9.9$ & 205 & Poco abundante & 8 \\
\hline $20-29.9$ & 77 & $10-14.9$ & 129 & Rara o escasa & 5 \\
\hline $30-39.9$ & 53 & $15-19.9$ & 44 & & \\
\hline $40-49.9$ & 29 & $20-24.9$ & 15 & & \\
\hline $50-59.9$ & 17 & $25-29.9$ & 5 & & \\
\hline $60-69.9$ & 4 & $30-34.9$ & 2 & & \\
\hline $70-79.9$ & 2 & $35-39.9$ & 1 & & \\
\hline $80-89.9$ & 2 & & & & \\
\hline $90-99.9$ & 0 & & & & \\
\hline $100-109.9$ & 1 & & & & \\
\hline $110-119.9$ & 2 & & & & \\
\hline $120-129.9$ & 3 & & & & \\
\hline $130-139.9$ & 1 & & & & \\
\hline $140-149.9$ & 0 & & & & \\
\hline $150-159.9$ & 0 & & & & \\
\hline $160-169.9$ & 1 & & & & \\
\hline $170-179.9$ & 1 & & & & \\
\hline $180-189.9$ & 0 & & & & \\
\hline $190-199.9$ & 1 & & & & \\
\hline $200-209.9$ & 0 & & & & \\
\hline $210-219.9$ & 2 & & & & \\
\hline Total & 410 & & 410 & & 17 \\
\hline
\end{tabular}


Cuadro 4. Estructura horizontal del bosque, a partir de la distribución de árboles por clase diamétrica y de altura, de las especies presentes en los transectos del área evaluada del BES Cerro Tres Puntas (Salas-Motupe, Lambayeque, Perú).

\section{Altura Diámetro}

Transecto Núm. individuos

(m)

\section{Media \pm ds $\quad$ Media \pm ds}

\begin{tabular}{lrrr}
\hline 1 & 110 & $10.21 \pm 4.35$ & $20.11 \pm 12.28$ \\
2 & 42 & $10.33 \pm 4.99$ & $21.65 \pm 12.52$ \\
4 & 194 & $9.58 \pm 3.74$ & $23.11 \pm 24.25$ \\
\hline
\end{tabular}

\section{Densidad}

La especie con el mayor número de individuos y, por lo tanto, con la más densidad relativa (DER) fue $V$. macracantha, con 154 (37.6\%), seguido de $C$. iguanaea con $55(13.4 \%)$ y Erythrina smithiana Krukoff. con 32 (7.8\%); por consiguiente, a Fabaceae $(59.4 \%)$ y Cannabaceae $(13.1 \%)$ les correspondieron los valores más altos; en tanto que, el taxon con el menor número de individuos fue $B$. sulcata $(0.2$ \%) (cuadros 1 y 4). La frecuencia relativa (FER) alcanzó el máximo valor (8.51) en varios taxones: V. macracantha, L. huasango, P. excelsum, A. multiflora, F. obtusifolia y C. iguanaea. V. macracantha, C. Iutea, E. smithiana y C. iguanaea se consideraron abundantes; ocho poco abundantes y cinco raras o escasas: $B$. graveolens, C. glabrata, E. ruizii, C. iguanaea y B. sulcata, esta última, con apenas un individuo (Cuadro 3). 


\section{Área basal}

El máximo valor de DAP correspondió a $B$. sulcata con $124.10 \mathrm{~cm}$, seguido por $F$. obtusifolia, $(120.06 \mathrm{~cm})$, C. iguanaea $(38.48 \mathrm{~cm})$ y P. limensis $(37.31 \mathrm{~cm})$; aunque, solamente se identificó un ejemplar de $B$. sulcata y ocho de $P$. limensis (Cuadro 1 ). Con excepción de la clase 5-9.9 cm DAP con 86 individuos, el análisis de la distribución diamétrica indicó que la mayoría de los individuos en los transectos se reunieron en las clases 10 - 19.9 cm DAP (205) y 20 - 29.9 cm DAP (129), cuya proporción disminuyó a medida que aumentaban los diámetros (Cuadro 3). El valor acumulado de área basal fue de $343.86 \mathrm{~m}^{2} \mathrm{ha}^{-1}$, conformada por B. sulcata $139.28 \mathrm{~m}^{2} \mathrm{ha}^{-1}$ y $F$. obtusifolia $\left(120.90 \mathrm{~m}^{2} \mathrm{ha}^{-1}\right)$. La mayor cobertura relativa (COB) o área basal relativa más amplia coincidió con las mismas especies (B. sulcata, con 39.19 y F. obtusifolia, con 36.68), seguidas de manera muy distante por C. iguanaea (3.77), P. limensis (3.54) y E. smithiana (3.30); y a nivel de familia destacaron Lauraceae (39.19), Moraceae (36.68) y Fabaceae (11.28) (Cuadro 5). 
Cuadro 5. Variables ecológicas de las especies presentes en el área evaluada del BES Cerro Tres Puntas (Salas-Motupe, Lambayeque, Perú).

\begin{tabular}{|c|c|c|c|c|c|c|c|c|}
\hline Familia/Especie & $\begin{array}{l}\text { Núm. } \\
\text { esp. }\end{array}$ & $\begin{array}{l}\text { Núm. } \\
\text { ind. }\end{array}$ & DER & FER & $\begin{array}{c}\text { Área basal } \\
\left(\mathrm{m}^{2}\right)\end{array}$ & $\begin{array}{c}\text { Área } \\
\text { Basal } \\
\text { relativa } \\
\text { (COB) }\end{array}$ & IVI & VIF \\
\hline \multicolumn{9}{|l|}{ Anacardiaceae } \\
\hline Loxopterygium huasango Spruce ex Engl. & & 20 & 4.88 & 8.51 & 6.06 & 1.97 & 15.35 & \\
\hline Total Familia & 1 & 20 & 4.88 & 8.51 & 6.06 & 1.97 & 15.35 & 12.73 \\
\hline \multicolumn{9}{|l|}{ Boraginaceae } \\
\hline Cordia lutea Lam. & & 29 & 7.07 & 6.38 & 0.92 & 0.30 & 13.74 & \\
\hline Total Familia & 1 & 29 & 7.07 & 6.38 & 0.92 & 0.30 & 13.74 & 13.27 \\
\hline \multicolumn{9}{|l|}{ Burseraceae } \\
\hline Bursera graveolens (Kunth) Triana \& Planch. & & 4 & 0.98 & 4.26 & 3.95 & 1.28 & 6.53 & \\
\hline Total Familia & 1 & 4 & 0.98 & 4.26 & 3.95 & 1.28 & 6.53 & 8.16 \\
\hline \multicolumn{9}{|l|}{ Fabaceae } \\
\hline Albizia multiflora (Kunth) Barneby \& J.W. Grimes & & 8 & 1.95 & 8.51 & 8.75 & 2.84 & 13.30 & \\
\hline Caesalpinia glabrata Kunth & & 5 & 1.22 & 2.13 & 1.11 & 0.36 & 3.71 & \\
\hline Erythrina smithiana Krukoff & & 32 & 7.80 & 6.38 & 10.19 & 3.30 & 17.49 & \\
\hline Pithecellobium excelsum (Kunth) Mart. & & 21 & 5.12 & 8.51 & 0.96 & 0.31 & 13.94 & \\
\hline Prosopis limensis Benth. & & 9 & 1.95 & 2.13 & 10.93 & 3.54 & 7.62 & \\
\hline Senna atomaria (L.) Irwing \& Barneby & & 15 & 3.66 & 4.26 & 0.76 & 0.25 & 8.16 & \\
\hline Vachellia macracantha (Willd.) Seigler \& Ebinger & & 154 & 37.56 & 8.51 & 2.09 & 0.68 & 46.75 & \\
\hline Total Familia & 7 & 244 & 59.40 & 40.50 & 34.79 & 11.28 & 110.97 & 111.86 \\
\hline \multicolumn{9}{|l|}{ Lauraceae } \\
\hline Beilschmiedia sulcata (Ruiz \& Pav.) Kosterm & & 1 & 0.24 & 2.13 & 120.90 & 39.19 & 41.57 & \\
\hline Total Familia & 1 & 1 & 0.24 & 2.13 & 120.90 & 39.19 & 41.57 & 45.33 \\
\hline \multicolumn{9}{|l|}{ Malvaceae } \\
\hline Eriotheca ruizii (K. Schum.) A. Robyns & & 6 & 1.46 & 2.13 & 9.74 & 2.49 & 6.80 & \\
\hline Guazuma ulmifolia Lam. & & 11 & 2.68 & 6.38 & 6.53 & 1.91 & 10.98 & \\
\hline Total Familia & 2 & 17 & 4.16 & 8.52 & 16.27 & 4.40 & 17.78 & 20.32 \\
\hline \multicolumn{9}{|l|}{ Moraceae } \\
\hline Ficus obtusifolia Kunth & & 17 & 4.16 & 8.51 & 139.28 & 36.68 & 49.34 & \\
\hline Total Familia & 1 & 17 & 4.16 & 8.53 & 139.28 & 36.68 & 49.34 & 46.74 \\
\hline \multicolumn{9}{|l|}{ Olacaceae } \\
\hline Ximenia americana L. & & 17 & 4.15 & 6.38 & 0.92 & 0.33 & 10.86 & \\
\hline Total Familia & 1 & 17 & 4.15 & 6.38 & 0.92 & 0.33 & 10.86 & 10.38 \\
\hline \multicolumn{9}{|l|}{ Sapindaceae } \\
\hline Sapindus saponaria L. & & 6 & 1.46 & 6.38 & 2.69 & 0.79 & 8.63 & \\
\hline Total Familia & 1 & 6 & 1.46 & 6.38 & 2.69 & 0.79 & 8.63 & 8.15 \\
\hline \multicolumn{9}{|l|}{ Cannabaceae } \\
\hline Celtis iguanaea (Jacq.) Sarg. & & 55 & 13.41 & 8.51 & 13.38 & 3.77 & 25.70 & \\
\hline Total Familia & 1 & 55 & 13.41 & 8.51 & 13.38 & 3.77 & 25.70 & 23.08 \\
\hline Total & 17 & 410 & 100 & 100 & 343.86 & 100 & 300 & 300 \\
\hline
\end{tabular}




\section{Índice de Valor de Importancia por Especie (IVI) e Índice de Valor de Importancia por Familia (VIF)}

El análisis del IVI de las 17 especies evaluadas destacó a $F$. obtusifolia (49.34) sobre V. macracantha (46.75) y C. ruiziana (41.57); a su vez, a Fabaceae (110.97), Moraceae (49.34) y Lauraceae (41.57) (Cuadro 5). Estas familias registraron el VIF más alto (Fabaceae, 111.86; Moraceae, 46.74 y Lauraceae, 45.33); mientras que, las de valor más bajo fueron Burseraceae (8.16) y Sapindaceae (8.15) (Cuadro 5).

\section{Discusión}

El área de muestreo corresponde a la clasificación de bosques estacionalmente secos de montaña en la costa norte que cubre los BES sobre los $500 \mathrm{msnm}$, y abarca los flancos occidentales de los Andes en las regiones de Tumbes, Piura y Lambayeque, hasta La Libertad, incluida parte de Cajamarca (Linares-Palomino y Pennington, 2007). Sin embargo, como lo indica La Torre-Cuadros y Linares-Palomino (2008) este límite altitudinal es bastante variable, discutible y depende de varios factores.

Dado que el muestreo inició en los 373 msnm, incorporó una pequeña área de Ilanura en la costa norte que cubre los BES debajo de los 500 msnm a lo largo de los flancos occidentales de los Andes, desde Tumbes hasta La Libertad (LinaresPalomino y Pennington, 2007). Esta sería la razón por la que en las condiciones de Pilasca se observó escasa presencia de $P$. limensis (algarrobo), solamente un espécimen de $C$. scabridum (zapote) y otros de Beautempsia avicennifolia (Kunth) Gaudich. (vichayo); estos últimos fuera del área de muestreo.

Los mismos autores indicaron, además, que los BES secos de montaña en la costa norte, al igual que los del sistema interandino del río Marañón son las más ricos en especies leñosas con 193 y 184, respectivamente. Esa información es corroborada con el estudio del bosque tropical estacionalmente seco (BTES) del Valle del Marañón, en el norte del Perú, en el que se identifican 440 taxa leñosos y 143 (33\%) endémicas, así como dos centros de endemismo dentro del Valle, con $56 \%$ de especies y $78 \%$ de los géneros identificados en los BTES peruanos (Marcelo-Peña et al., 2016). 
En el análisis de los patrones de diversidad de las especies (4 660 leñosas) de los BTES de América Latina y el Caribe, se determinó que la notable rotación florística observada implica la necesidad de establecer múltiples áreas de conservación para proteger esa importante diversidad florística (DRYFLOR et al., 2016).

A pesar de que una lista anotada de los BES del Ecuador y otros estudios relacionados (Svenson, 1946; Gentry, 1995b; Aguirre et al., 2006) señalaron que los BES de ambos países, en especial los de la costa, formaban una sola unidad geográfica, con alto número de taxones endémicos (Centro de Endemismo Tumbesino). Las pocas especies (17), géneros (17) y familias (10) inventariadas en el Cerro Tres Puntas de Pilasca demostrarían un alto grado de depredación de la flora original; ello corroboraría lo observado en otros BES, como Chaparrí en Lambayeque (Linares-Palomino y Ponce-Álvarez, 2009; Martos et al., 2009) y La Menta y Timbes en Piura (Rasal et al., 2011).

Por otro lado, habría que considerar otros factores, como los gradientes de diversidad y densidad de especies, así como de precipitación que disminuyen conforme los BES se extienden hacia el sur, y que incluyen a las regiones de Lambayeque y La Libertad. Por la agreste y peligrosa topografía del terreno, el muestreo lineal que se utilizó en la presente investigación hizo posible evaluar un área extensa que abarcó 11.4 ha, muestreo que se ha usado en los inventarios botánicos rápidos (IBR) en los que se aborda la vegetación leñosa, endemismos y el estado de conservación de los BES de Jaén (Perú) (Marcelo-Peña, 2008), con base en la metodología de Hawthorne y Abu-Juam (1995) y Gordon et al. (2004).

A pesar de que en los últimos años el interés por los BES se ha incrementado (Espinosa et al., 2012; Aguirre y Geada-López, 2017), en los escasos trabajos efectuados en Ecuador y Perú, se observó que los métodos de muestreo fueron muy variados entre el uso de los transectos rectangulares y el muestreo lineal, así como en el tamaño de las unidades muestrales, desde 0.25 hasta 6.5 hectáreas; y en el DAP de las especies muestreadas, de $\geq 1.0$ a $10 \mathrm{~cm}$. A esto habría de agregarse la diferencia entre el número de individuos, especies, géneros y familias que suele ser más alta en áreas naturales protegidas como el Parque Nacional Cerros de Amotape (Linares-Palomino, 2005) y muy 
deprimida en otras áreas históricamente depredadas, como la Reserva Ecológica Chaparrí (Linares-Palomino y Ponce-Álvarez, 2009).

De todos ellos, el que más se aproximó al aquí descrito es el de La Menta y Timbes en Piura (Rasal et al., 2011), donde no obstante que se registraron especies leñosas con DAP $\geq 1.0 \mathrm{~cm}$, el número de individuos inventariados fue significativamente menor, aunque hubo bastante similitud en el número de especies, géneros y familias.

En su estudio de la Reserva Ecológica Chaparrí, Linares-Palomino y Ponce-Álvarez (2009) puntualizaron la dificultad de establecer comparaciones directas de inventarios en los bosques estacionalmente secos de la región, debido a la gran variación entre las metodologías de muestreo utilizadas, pisos altitudinales, área total de muestreo, DAP de los especímenes muestreados, estado de conservación y de depredación del área en el momento de su realización, e incluso en la priorización de la información recolectada, entre otros factores.

Lo anterior se confirma con los resultados de varios estudios en Perú; por ejemplo, en los bosques de Jaén, Cajamarca (Marcelo-Peña, 2008); La Menta y Timbes en Ayabaca, Piura (Rasal et al., 2011); Reserva de la Biosfera del Noroeste del Perú (Parque Nacional Cerros de Amotape y Zona Reservada de Tumbes) (Leal-Pinedo y Linares-Palomino, 2005); Llanuras Costeras de Piura (La Torre-Cuadros y Linares-Palomino, 2008); Parque Nacional Cerros de Amotape (Linares-Palomino, 2005); y Reserva Ecológica Chaparrí, Lambayeque (Linares-Palomino y PonceÁlvarez, 2009). Sin embargo, no ha sido posible establecer patrones rigurosos de comparación entre toda esta valiosa información y el estudio desarrollado en el Cerro Tres Puntas de Pilasca.

En las formaciones vegetales de los BES de Ecuador y Perú se elaboraron listas de numerosas especies leñosas para el bosque deciduo, bosque seco semideciduo mezclado con especies siempre verdes, bosque caducifolio y el matorral espinoso seco (Aguirre et al., 2006; Aguirre y Kvist, 2009); No obstante, en el trabajo aquí descrito, solamente, se registraron ocho especies: $P$. limensis, $C$. lutea, $P$. excelsum, C. glabrata, V. macracantha, E. smithiana, B. graveolens y $L$. huasango, lo que, de alguna manera, indicaría la fuerte presión antrópica a la que está sujeta la vegetación del Cerro Tres Puntas de Pilasca en los últimos años, aun cuando fue posible encontrar individuos esporádicos de 
Bougainvillea peruviana Nees \& Mart., Bauhinia aculeata L., C. vitifolium y C. scabridum; aunque estaban fuera del transecto lineal.

La tendencia de distribución de las alturas, que no se ajusta exactamente con la "J" invertida, descrita por Lamprecht (1990) y Günter et al. (2011) reflejaría la característica sucesional del bosque debido a los disturbios constantes y selectivos que ejercen los habitantes en el lugar, manteniéndolo en una sucesión secundaria (Rasal et al., 2012). Aunque en la experiencia referida no se incluyeron individuos con DAP < $5.0 \mathrm{~cm}$, pero sí con altura de planta $<4.9 \mathrm{~m}$, los resultados permiten alcanzar un cabal conocimiento sobre el proceso de regeneración de las especies presentes en las clases diamétricas y de altura mayores, como fue recomendado por García et al. (2010) al estudiar la estructura y diversidad florística de dos bosques naturales en el Cauca, Colombia.

En los BES La Menta y Timbes de Piura (Perú), alrededor de 55.0 \% de los individuos muestreados tuvieron un DAP entre $0-10.0 \mathrm{~cm}$ con algunos individuos de L. huasango y B. graveolens con DAP entre 50-60 cm, para La Menta; y Ceiba trischistandra Bakh. y E. ruizii con DAP entre $80-90 \mathrm{~cm}$, para Timbes (Rasal et al., 2011). Los resultados que se presentan para el BES Cerro Tres Puntas de las cinco especies indicadas solo se registró E. ruizii. Asimismo, de 410 individuos inventariados, 196 tuvieron DAP $\geq 20.0 \mathrm{~cm}$, y tres especies: V. macracantha, C. iguanaea y E. smithiana contribuyeron con $58.8 \%$ del total cuantificado.

En muy pocos estudios de los bosques secos del Perú se ha recabado información o se han efectuado análisis relacionados con AB (Área Basal), IVI (Índice de Valor de Importancia) y VIF (Valor de Importancia para la Familia); sin embargo, Linares-Palomino y Ponce-Álvarez (2009) calcularon un $A B$ de $11.74 \mathrm{~m}^{2}$, en el que sobresalieron E. ruizii y $L$. huasango con 4.40 y $4.12 \mathrm{~m}^{2}$, respectivamente en la Reserva Ecológica de Chaparrí (Lambayeque). En los bosques secos de La Menta y Timbes (Piura), Rasal et al. (2011) obtuvieron, para La Menta, un AB de $128.86 \mathrm{~m}^{2}$, en donde destacaron L. huasango $\left(45.97 \mathrm{~m}^{2}\right)$ y B. graveolens (34.69 $\mathrm{m}^{2}$ ); en Timbes se obtuvieron un $A B$ de $196.63 \mathrm{~m}^{2}$, con especial aportación de E. smithiana $\left(47.16 \mathrm{~m}^{2}\right)$ y $C$. trischistandra $\left(38.88 \mathrm{~m}^{2}\right)$. En ambos casos, el $A B$ fue muy inferior al estimado en el presente estudio, cuya AB fue de $343.86 \mathrm{~m}^{2} \mathrm{ha}^{-1}$, lo 
que indicó que a pesar de que en el bosque Cerro Tres Puntas existe una fuerte presión antrópica, esta no ha resultado tan dramática como ocurre en Chaparrí, La Menta y Timbes, donde incluso al bosque de Chaparrí se le definió como una versión empobrecida de los bosques estacionalmente secos de la región (LinaresPalomino y Ponce-Álvarez, 2009).

En esta misma comunidad, cuatro especies alcanzaron valores de IVI superiores a 30.0: E. ruizii (58.22), L. huasango (44.93), C. lutea (33.76) y B. graveolens (30.08), que correspondieron a las familias Malvaceae, Anacardiaceae, Boraginaceae y Burseraceae, respectivamente (Linares-Palomino y Ponce-Álvarez, 2009); en tanto que, en La Menta, los valores más altos fueron para B. graveolens (50.0), L. huasango (43.0) y C. lutea (36.0); y en Timbes, a C. lutea (62.0), E. smithiana (40.0) y C. trischistandra (25.0) (Rasal et al., 2011). Asimismo, como se ha referido anteriormente, en el trabajo de los bosques secos en la provincia de Loja, Aguirre et al. (2013) determinaron las cinco especies con el IVI más alto: $C$. trischistandra, Simira ecuadorensis (Standl.) Steyerm., Tabebuia chrysantha G. Nicholson, E. ruizii y Terminalia valverdeae A. H. Gentry; aunque ninguna información cuantitativa fue proporcionada. Al comparar esos resultados con los que aquí se documentan, se observan diferencias notorias; puesto que las especies que destacaron ampliamente fueron $F$. obtusifolia (49.34), V. macracantha (46.75) y $B$. sulcata (41.57), las cuales fueron relevantes en otros bosques estacionales. Es posible enunciar la hipótesis de que la depredación de las especies en estos bosques no tiene un patrón definido, y ello habría estado supeditado a la preferencia por las especies maderables más valiosas, así como a la misma protección que les habrían dado los pobladores del lugar, por lo que, ciertamente, en el Cerro Tres Puntas la depredación ha sido más selectiva.

Los resultados del VIF de la investigación descrita para el Cerro Tres Puntas de Pilasca en la que sobresalieron las familias Fabaceae (111.86), Moraceae (46.74) y Lauraceae (45.33), discrepan ampliamente de las referidas para Chaparrí (Linares-Palomino y Ponce-Álvarez, 2009), La Menta y Timbes (Rasal et al., 2011); ya que ninguna de las familias consignadas en esos trabajos fue identificada en el estudio realizado en el área de interés. 


\section{Conclusiones}

El estudio realizado en el Cerro Tres Puntas de Pilasca (Salas-Motupe, Lambayeque) contribuye al conocimiento de los ecosistemas naturales del Perú, específicamente, el de los bosques estacionalmente secos, ya que determina su estructura y composición florística. En el contexto de la biodiversidad, los resultados describen su riqueza vegetal, lo que es una aportación valiosa para efectos de conservación y manejo de dichos recursos, y los datos ecológicos constituyen una aproximación preliminar a la dinámica de los BES de la región referida.

Se sugiere ampliar los estudios a otras áreas en el mismo Cerro Tres Puntas, en áreas geográficas adyacentes, en otros BES de Lambayeque como los de Tongorrape, Naupe y Tocmoche e incluso incrementar el intervalo altitudinal del muestreo. Asimismo, orientar las evaluaciones sobre captura de carbono e identificar las especies que más contribuirían a mitigar el cambio climático para propender su multiplicación a gran escala con fines de reforestación.

\section{Agradecimientos}

Los autores agradecen al Vicerrector de Investigación de la Universidad Nacional Pedro Ruiz Gallo (UNPRG) de Lambayeque, Dr. Ernesto Hashimoto Moncayo, por las facilidades otorgadas en la ejecución del presente estudio, así como al personal administrativo de la UNPRG: Héctor Valdiviezo Castillo y Melissa J. Saldarriaga Heros y a la Br. Wendy Herrera Moncada, por la asistencia técnica en la elaboración del manuscrito. Asimismo, a los comuneros de Pilasca, Froilán Sánchez e Isabel Valencia, por sus invalorables servicios de guía en los varios ascensos realizados en el Cerro Tres Puntas.

\section{Conflicto de intereses}

Los autores declaran no tener conflicto de intereses 


\section{Contribución por autor}

Guillermo E. Delgado Paredes: planeación y supervisión del proyecto, redacción del manuscrito; Cecilia Vásquez Díaz: toma de datos de campo; Fernando Tesén Núñez: toma de datos de campo; Boris Esquerre Ibáñez: toma de datos de campo y apoyo en el análisis de datos; Felipe Zuñe Da Silva: análisis de datos y revisión del manuscrito; Consuelo Rojas-Idrogo: planeación del proyecto, redacción y revisión del manuscrito.

\section{Referencias}

Aguilar, P. 1994. Características faunísticas del norte del Perú. Arnaldoa 2(1):77-102.

Aguirre, Z., R. Linares P. y L. P. Kvist. 2006. Especies leñosas y formaciones vegetales en los bosques estacionalmente secos de Ecuador y Perú. Arnaldoa 13(2):324-350. ISSN: 1815-8242.

Aguirre, Z. y L. Kvist. 2009. Composición florística y estructura de bosques estacionalmente secos en el sur-occidental de Ecuador, Provincia de Loja, municipios de Macará y Zapotillo. Arnaldoa 16(2):87-99. Doi: 10.13140/2.1.1288.0008.

Aguirre, Z., Y. Betancourt y G. Geada. 2013. Composición florística y estructura de los bosques secos de la Provincia de Loja, Ecuador. Arnaldoa 20:117-128. ISSN: $1815-8242$

Aguirre, Z. y G. Geada-López. 2017. Estado de conservación de los bosques secos de la provincia de Loja, Ecuador. Arnaldoa 24(1):207-228.

Doi:10.22497/arnaldoa.241.24107.

Angiosperm Phylogeny Group III (APG III). 2009. An update of the Angiosperm Phylogeny Group classification for the orders and families of flowering plants: AGP III. Botanical Journal of the Linnean Society 161:105-121.

Doi: 10.1111/j.1095-8339.2009.00996.x. 
Angiosperm Phylogeny Group IV (APG IV). 2016. An update of the Angiosperm Phylogeny Group classification for the orders and families of flowering plants: APG IV. Botanical Journal of the Linnean Society 181:1-20.

Doi:10.1111/boj.12385.

Brack, A. y C. Mendiola. 2004. Ecología del Perú. PNUD. Asociación Editorial Bruño. Lima, Perú. 495 pp.

Dryflor, K. Banda-R, A. Delgado-Salinas, K. G. Dexter, R. Linares-Palomino, A. Oliveira-Filho, D. Prado, M. Pullan, C. Quintana, R. Riina, G.M. Rodriguez M., J. Weintritt, P. Acevedo-Rodriguez, J. Adarve, E. Álvarez, A. Aranguren B., J. Camilo Arteaga, G. Aymard, A. Castaño, N. Ceballos-Mago, Á. Cogollo, H. Cuadros, F. Delgado, W. Devia, H. Dueñas, L. Fajardo, Á. Fernández, M. A. Fernández, J. Franklin, E. H. Freid, L. A. Galetti, R. Gonto, R. González-M., R. Graveson, E. H. Helmer, Á. Idárraga, R. López, H. Marcano-Vega, O. G. Martínez, H.M. Maturo, M. MacDonald, K. Maclaren, O. Melo, F. Mijares, V. Mogni, D. Molina, N. P. Moreno, J. M. Nassar, D. M. Neves, L. J. Oakley, M. Oatham, A. R. Olivera-Luna, F. F. Pezzini, O. J. Reyes Dominguez, M. E. Rios, O. Rivera, N. Rodriguez, A. Rojas, T. Sarkinen, R. Sánchez, M. Smith, C. Vargas, B. Villanueva and R.T. Pennington. 2016. Plant diversity patterns in neotropical dry forests and their conservation implications. Science 353(6306): 1383-1387. Doi: 10.1126/science.aaf5080.

Espinosa, C., M. De la Cruz, A. L. Luzuriaga y A. Escudero. 2012. Bosques tropicales secos de la región Pacífico Ecuatorial: diversidad, estructura, funcionamiento e implicaciones para la conservación. Ecosistemas 21(1-2):167-179.

García, C., C. Suárez y M. Daza. 2010. Estructura y diversidad florística de dos bosques naturales (Buenos Aires, Dpto. Cauca, Colombia). Revista Biotecnología en el Sector Agropecuario y Agroindustrial (Universidad del Cauca) 8(1):74-82.

Gentry, A. H. 1982. Patterns of neotropical diversity. Evolutionary Biology 15:184. Doi: 10.1007/978-1-4615-6968-8_1. 
Gentry, A. H. 1995a. Patterns of diversity and floristic composition in Neotropical Montane Forests. In: Churchill, S. P., H. Balslev, E. Forero and J. L. Luteyn (eds.). Biodiversity and Conservation of Neotropical Montane Forests. The New York Botanical Garden. New York, NY, USA. pp. 103-126.

Gentry, A. H. 1995b. Diversity and floristic composition of neotropical dry forests. In: Bullock, S. H., H. A. Mooney and E. Medina (eds.). Seasonally Dry Tropical Forests. Cambridge University Press, Cambridge, UK. pp. 146-194.

Gobierno Regional de Lambayeque. 2013. Estudio Geológico del Departamento de Lambayeque. Lambayeque, Perú. 60 p.

Google Earth. 2019. Salas, Perú. 17M657260.19 m E9312897.45 m S, Altitud del ojo 11.65 km. CNES/Airbus 2019. http://www.earth.google.com (2 de diciembre, 2019).

Gordon, J. E., W. D. Hawthorne, A. Reyes-García, G. Sandoval and A. J. Barrance. 2004. Assesing landscapes: a case study of tree and shrub diversity in the seasonally dry tropical fortest of Oaxaca, Mexico and southern Honduras. Biological Conservation 117:429-442. Doi:10.1016/j.biocon.2003.08.011.

Günter, S., M. Weber, B. Stimm and R. Mosandl (eds.). 2011. Silviculture in the Tropics. Tropical Forestry 8. Springer-Verlag. Berlin, Germany. 560 p.

Hawley, R. C. y D. M. Smith. 1972. Silvicultura Práctica. Ediciones Omega. Barcelona, España. 544 p.

Hawthorne, W. D. and M. Abu-Juam. 1995. Forest protection in Ghana. With particular reference vegetation and plant species. Forest Inventory and Management Planning Brach. Forestry Department Kumasi, Ghana. IUCN/ODA/Forest Department, Republic of Ghana. https://www.iucn.org/es/content/forest-protection-ghana-particular-referencevegetation-and-plant-species (20 de septiembre de 2019). 
Lamprecht, H. 1990. Silvicultura en los trópicos. Deutsche Gesellschaft fur Technische Zusammenartbeit (GTZ). Alemania. 334 p.

La Torre-Cuadros, M. A. y R. Linares-Palomino. 2008. Mapas y clasificación de vegetación en ecosistemas estacionales: un análisis cuantitativo de los bosques secos de Piura. Revista Peruana de Biología 15(2):31-42. Doi: 10.15381/rpb.v15i1.1668.

Leal-Pinedo, J. M. y R. Linares-Palomino. 2005. Los bosques secos de la Reserva de Biosfera del Noroeste (Perú): Diversidad arbórea y estado de conservación. Caldasia 27(2):195-211.

Linares-Palomino, R. 2005. Spatial distribution patterns of trees in a seasonally dry forest in the Cerros de Amotape National park, nortwestern Peru. In: Weigend, M., E. Rodríguez and C. Arana. (comps.). Bosques Relictos del NO de Perú y SO de Ecuador. Revista Peruana de Biología 12:317-326.

Linares-Palomino, R. 2006. Phytogeography and floristics of seasonally dry tropical forests in Peru. In: Pennington, R. T., G. P. Lewis and J. A. Ratter (eds.). Neotropical Savannas and Seasonally Dry Forests: Plant Diversity, Biogeography and Conservation. CRC Press. Boca Raton, FL, USA. pp. 257-279. Doi: 10.1201/9781420004496.

Linares-Palomino, R. and S. I. Ponce-Álvarez. 2009. Structural patterns and floristics of a seasonally dry forest in Reserva Ecológica Chaparrí, Lambayeque, Perú. Tropical Ecology 50:305-314.

Linares-Palomino, R. y R. T. Pennington. 2007. Lista anotada de plantas leñosas en bosques estacionalmente secos del Perú - una nueva herramienta en internet para estudios taxonómicos, ecológicos y de biodiversidad. Arnaldoa 14(1):149-152.

López, M. 2013. Geomorfología y Geología Histórica de Lambayeque. https://www.academia.edu/14393809/Geomofología_de_Lambayeque. (15 de enero de 2019).

Maleheiros, D. E. O. y E. Rotta. 1982. Levantamiento horizontal de uma mata de araucaria do primero planalto paranaense. Boletim de Pesquisa. Florianópolis 4:1-39. 
Marcelo-Peña, J. L. 2008. Vegetación leñosa, endemismos y estado de conservación en los bosques estacionalmente secos de Jaén, Perú. Revista Peruana de Biología 15:43-52. Doi: 10.15381/rpb.v15i1.1669.

Marcelo-Peña, J. L., I. Huamantupa, T. Särkinen and M. Tomazello. 2016. Identifying conservation priority areas in the Marañón valley (Peru) based on floristics inventories. Edinburgh Journal of Botany 73:95-123.

Doi:10.1017/S0960428615000281.

Martos, J. R., M. Scarpati, C. Rojas y G. E. Delgado. 2009. Fenología de algunas especies que son alimento para la pava aliblanca Penelope albipennis. Revista Peruana de Biología 15: 51-58. Doi: 10.15381/rpb.v15i2.1721.

Miles, L., A. C. Newton, R. S. Defries, C. Ravilious, I. May, S. Blyth, V. Kapos and J. E. Gordon. 2006. A global overview of the conservation status of tropical dry forests. Journal of Biogeography 33:491-505. Doi: 10.1111/j.13652699.2005.01424.x.

Ministerio del Ambiente (Minam). 2019. Mapa Nacional de Ecosistemas del Perú. Lima, Perú. 60 p.

Rangel, J. O. y A. Velásquez. 1997. Métodos de estudio de la vegetación. In: Rangel, J. O., P. Lowy y M. Aguilar (eds.). Colombia Diversidad Biótica II. Instituto de Ciencias Naturales, Universidad Nacional de Colombia. Bogotá, Colombia. pp. 59-82.

Rasal, M., J. Troncos, C. Lizano, O. Parihuamán, D. Quevedo, C. Rojas y G. Delgado. 2011. Características edáficas y composición florística del bosque estacionalmente seco La Menta y Timbes, región Piura, Perú. Ecología Aplicada 10(2):61-74. Doi: 10.21704/rea.v10i1-2.414.

Rasal, M., J. Troncos, C. Lizano, O. Parihuamán, D. Quevedo, C. Rojas y G. Delgado. 2012. La vegetación terrestre del bosque montano de Lanchurán (Piura, Perú). Caldasia 34(1):1-24. 
Sagástegui, A., M. O. Dillon, I. Sánchez, S. Leiva y P. Lezama. 1999. Diversidad Florística del Norte del Perú. Tomo I. Editorial Graficart. Trujillo, Perú. 228 p.

Shepherd, G. J. 2010. Fitopac 2.1.2: Manual do usuario. UNICAMP. Campinas, São Paulo, Brasil. 93 p.

Servicio Nacional de Meteorología e Hidrología (Senamhi). 2018. Estación COMeteorológica Jayanca (LA Vic). Lambayeque, Perú. 4 p.

Steffen, W., A. Persson, L. Deutsch, J. Zalasiewicz, M. Williams, K. Richardson, C. Crumley, P. Crutzen, C. Folke, L. Gordon, M. Molina, V. Ramanathan, J. Rockström, M. Scheffer, H. J. Schellnuber and U. Svedin. 2011. The anthropocene: from global change to planetary stewardship. Ambio 40:739-761. Doi:10.1007/s1328 0-011-0185-x.

Svenson, H. K. 1946. Vegetation of the coast of Ecuador and Peru and its relation to the Galapagos Islands. I. Geographical relations of the flora. American Journal of Botany 33(5):394-426. Doi: 10.2307/2437128.

Weberbauer, A. 1922. Die Vegetationskarte der peruanischen Anden zwischen $5^{\circ}$ und $17^{\circ} \mathrm{S}$. Petermanns Geographische Mitteilungen 68:89-91.

Weberbauer, A. 1930. Die Pflanzendecke Nordperus im Departamento Tumbez una angrenzenden Teilen des Departamento Piura (3030' $-5^{\circ} \mathrm{s}$. Br.). Botanische Jahrbücher 63:29-48.

Weberbauer, A. 1936. Phytogeography of the Peruvian Andes. Field Museum of Natural History, Botanical Series 13:13-81.

Weberbauer, A. 1945. El mundo vegetal de los Andes peruanos. Ministerio de Agricultura, Dirección de Agricultura, Estación Experimental Agrícola de La Molina, Lima. Lima, Perú. 776 p.

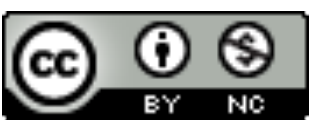

Todos los textos publicados por la Revista Mexicana de Ciencias Forestales - sin excepción- se distribuyen amparados bajo la licencia Creative Commons 4.0 Atribución-No Comercial (CC BY-NC 4.0 Internacional), que permite a terceros utilizar lo publicado siempre que mencionen la autoría del trabajo y a la primera publicación en esta revista. 